\section{Qualitative Researches In Social Sciences}

Prakash Upadhyay, Vikash Kumar KC

\section{Abstract}

Qualitative social science research is fundamentally embedded in grounded theory concerned with how the social world is interpreted, realized, understood and experienced, or produced. Qualitative investigation seeks answers to their questions in the realistic world. They congregate what they see, hear and read from the people and places and from events and activities and their main purpose are to learn about some aspects of the social world and to generate new understandings that can be used by that social world. The main objective of this study is the interpretation of social world especially of cultures and people's life-ways rather than seeking causal explanations for social-cultural practices. Nevertheless, in very rapidly changing information dominated globalized world, innovative traditions of the perception of emerging local and global contexts and realities need to be exposed and accepted as well as practiced in qualitative social science research.

Key Words : empirical, ethnographic, holographic, heuristic, inductive, iterative, rapport

\section{Backgroud}

Social Sciences study human being in the changing social and cultural context. Social and cultural contexts confront serious social, economic, political, ecological and other problems, which call for methodological, intelligent researching about their causes and also for finding out their remedies. As problems are related to human beings who are both unpredictable and undependable in behavior, consequently diverse methods are prerequisite for understanding and solving the problems and finding out the truth. To resolve diverse problems related to society and to satisfy the thirst of knowledge, social science research is prerequisite and for conducting a good social research it is indispensable to pursue a balanced social research method. Social research methods diverge along quantitative/qualitative dimensions in a rapidly altering world. Society and cultures are prototypes of human behavior and knowledge that every human discovers as a member of a changing society. Performing everything that engrosses comprehending human social, cultural, economic, political and other behaviors which are intangible and qualitative are the ingredients of social science studies which are primarily qualitative and hence social researchers are the observers who collect factual empirical information about people by watching, listening and interpreting what others are doing at micro level at the plebs level. Allied candidly with people's know-how, outlooks, and interpretations of the situations qualitative data is uniquely extracted from the heart and mind of the people. Interpretation is the major building block of qualitative research which is unswervingly related to people's experiences. Barnard et.al (2004) argues that qualitative data deals directly with people's experiences. It is grounded in a philosophical position, which is broadly Interpretivist concerned about how the social world is interpreted, understood and experienced, or produced. Qualitative researchers seek answers to their questions in the real world. They gather what they realize, see, hear and read from the people and places and from events and activities and the main intention are to learn about some aspects of the social world and to engender new understandings that can be used by that social world. Qualitative designs accentuate understanding of social phenomena through direct observation, communication with participants, or analysis of texts, and may stress contextual subjective accuracy over generality. In contrast quantitative research in social sciences are hectic more with extracting and analyzing statistical data that occurs numerically and are more tangible more precise, concrete and substantial. Quantitative approach explores social phenomena through quantifiable evidence, and often relies on statistical analysis to create valid and reliable general claims. The key objectives of this article are to sketch out the peculiarities and customary traditions of qualitative and quantitative studies and the process of qualitative data analysis.

\section{Data and Methods}

This article is based on secondary data which were obtained from various literatures, books, journals, booklets, articles etc.

\section{Qualitative and Quantitative Methods}

Concepts, theoretical perspectives, methods, tools and techniques differ sharply in qualitative and quantitative studies. Quantitative and the qualitative approaches have their flaw as well as potency. Both qualitative and quantitative classification is based on three groupings-

a. Study purpose,

b. Measurements of variables and

c. Analysis of information

The study is classified as qualitative if the purpose of the study is primarily to depict a situation, phenomenon, problem or event, the information is gathered through the use of variables measured on nominal scale (good, better, satisfactory, poor, etc.), or ordinal scale (first, second, third, etc.) i.e. qualitative measurement scales; if the analysis is done to establish the variation in the situation, phenomenon or problem without quantifying it. E.g. the description of an observed situation, the historical enumeration of events, and account of different opinions people have about an issue.

Methods selection with their tools and techniques depends upon the main purpose of research, discipline and theoretical stance. Both qualitative and quantitative methods observe different realities or different aspects of reality nevertheless the combination of these two methods will lead to a very balanced and high quality research. The divergences between qualitative and quantitative are immense. For example qualitative methods are less objective as they rely on qualitative data, scenario is based on natural settings with search for meaning, rejection of natural science; stand on idealist perspective with identification of cultural patterns. Qualitative methods are based on Inductive approaches. On the other hand, quantitative methods are more empirical as they are based on statistical data with the provision of experimental settings, adoption of natural science, pursuance of scientific laws with realist perspective and deductive approaches. Interpretive tradition of qualitative method in social sciences deals with the systematic approach signifying the art of asking, listening, and interpreting. The methods of collecting qualitative data can be grouped into four consistent groupings; methods, researcher's role, techniques and study site tradition are: 
Janapriya Journal of Interdisciplinary Studies, Vol. III (December 2014)

\begin{tabular}{|llll|}
\hline Methods & Researcher's role & Technique of data collection & Study site tradition \\
& & & \\
Observation & Observer & Participant observation & Field work tradition \\
In-depth interview & Interviewer & In-depth (key informant interview) & Field work tradition \\
Group discussion & Group moderator & Focus group discussion & Field work tradition \\
\hline
\end{tabular}

Qualitative research in social sciences are heuristic (discovery oriented) in nature based more on trial-and-error procedure for solving problems (or reaching an unclear goal) through incremental exploration, and by employing a known criteria to unknown factors. In social sciences researches, a well known heuristic is asking Who? What? When? Where? Why? while investigating a problem. A heuristic social researcher employs independent discovery, and relies heavily on common sense sense, creativity, and learning from experience. Unlike an algorithm (in quantitative studies), however, heuristic qualitative studies offers no guaranty of solving any problem. The heuristic Interpretivist tradition's chief dictum of qualitative methodology is to get people to open up and let them express themselves in their own terms and at their own pace in their own way. The prime issue is going among the people and building rapport for conducting a lofty research. The meaning of rapport is establishing harmonious relationship, togetherness, agreement, affinity, sympathy, concord empathy. Upadhyay (2011) alleges it connote winning the heart and mind of the people on whom the researcher's research is targeted on. It is done through rigorous and intensive field work tradition. Ethnographic researches are the excellent example of qualitative method. Sherpas through their rituals (1978) is a qualitative ethnographic works in/on Nepal by Sherry Ortner. Ortner conducted her rigorous field study in Dzemu 'Solu' of Nepal from September 1966 to February 1968. She collected primary data through field work by means of methods like ethnography, group discussion, interview, and observation. Her theoretical base was ritualistic approach.

Ortner made use of an assortment of diverse anthropological approaches that she expose during her research and puts into practice in her fieldwork and analysis. Although many approaches are used, of particular importance to her ethnography are the theoretical approaches of structuralism and symbolic analysis in a qualitative manner, which although is used particularly in interpretive approaches has been applied to various theoretical frameworks. This tendency towards symbolic analysis is evident by Ortner's stated view that culture is always biased, selected, partial and interpreted. This is also evident in the use of semantic (meanings and words of sentence) structure analysis and the focus on symbols as a way of imposing meaning. The theoretical approach that is the focus of analysis is structuralism; in particular this close scrutiny is achieved by focusing on the exemplary paradigmatic set and the role of ritual as a mediator and reconciler between contrasting aspects of society (Ortner 1986). By giving fastidious attention to certain similarities that bear a resemblance to each other within mysterious religious rites and symbolism portrayed in the ethnography it is probable to visibly interpret and understand the way that structural analysis can, and indeed been employed to access the communicative code of the Sherpa society. While interpreting and analyzing the symbols of Sherpa rituals, Sherry Ortner leads us toward the detection of conflict, contradiction, and stresses in the wider social and cultural world--- the best examples of interpretivist tradition in qualitative anthropological ethnographic study. Following a general ethnographic sketch a succinct description and interpretation of a ritual has been made by Ortner. The ritual has been sliced up, and its symbolic elements used as guides in the exploration and interpretation of problematic structures, relationships, and ideas of the Sherpa culture. Nyungne, exorcisms and offering rituals has been analyzed and interpreted by Ortner and are the case of hospitality (secular event) in order to present the network of Sherpa culture.

\section{The Pecularities and Customary Traditions}

There prevail the customary peculiarities and differences in qualitative and quantitative research methods. For example, quantitative and qualitative research methods are most often associated with deductive and inductive approaches, respectively. It means the two major types of reasoning in social science research are deduction and induction. Deductive research begins with known theory and tests it, usually by attempting to provide evidence for or against a pre-specified hypothesis. Inductive research begins by making observations, usually in order to develop a new hypothesis or contribute to new theory. Quantitative research usually follows the deductive and qualitative follow inductive. In quantitative methods attempts would be to test the hypothesis and /or prove or disprove the theory. The qualitative research on the other hand, builds theory moving from observations and open questions to more general conclusions. In other words, qualitative research applies inductive process to draw conclusion.

For example let's take the following cases from Nepal.....

A. If Shyam belongs to one of the political party in Nepal, then he is in favor of promulgating constitution.

He belongs to UML (United Marxist-Leninist) party. Hence, he is in favor of promulgating constitution.

B. Shyam and Nisha are affiliated with UML. Ram and Sita are associated with NC (Nepali Congress), and Bibek and Sharmila are associated with United Maoist party. They all are in favor of promulgating constitution.

Hence, all parties are in favor of drafting constitution.

Case "A" exemplifies deductive reasoning and case "B" exemplifies inductive reasoning.

Quantitative research is usually linked to the notion of science as objective truth or fact, whereas qualitative research is more often identified with the view that science is live experience and therefore subjectively determined. Quantitative research usually begins with pre-specified objectives focused on testing preconceived outcomes. Qualitative research usually begins with open-ended observation and analysis, most often looking for patterns and processes that explain how and why questions.

When applying quantitative methods, numerical estimation and statistical inference from a generalizable sample are often used in relation to a larger true population of interest. In qualitative research, narrative description and constant comparison are usually used in order to understand the specific populations or situations being studied. As a result, quantitative research is most often seen as a method trying to demonstrate causal relationships under standardized (controlled) conditions. Conversely, qualitative research is usually seen as a method seeking better understanding of some particular, natural (uncontrolled) phenomenon.

The nature of the general theoretical debate is characterized by fundamentally different understandings or beliefs about scientific research, in particular, and the world, in general. Adherence to different and separate paradigms can trap researchers into believing that there is only one true "scientific" way to conduct research. Exceptions to the general rules or doctrines allied with these research approaches suggest that many of the clashes between researchers' perspectives are more a question of belief systems and mutual lack of understanding than of methods. Nonetheless, the arguments continue to focus on methodological aspects 


\section{Grounded Theory in Qualitative Research}

Grounded theory is one of four qualitative designs commonly used in the human and social sciences; the other designs are ethnographies, case studies, and phenomenological studies. The key discrepancy between grounded theory and the other designs is the stress on theory development (Denzin \& Lincoln, 2005). Grounded theory holds the notion that theory could materialize through qualitative data analysis. In grounded theory, the researcher uses multiple stages of collecting, refining, and categorizing the data. Making constant comparisons and applying theoretical sampling are necessary strategies used for developing grounded theory (Creswell, 2007).

Closely associated to grounded theory, the constant comparative method is used to develop concepts from the data by coding and analyzing at the same time (Taylor \& Bogdan, 1998). The constant comparative method "combines systematic data collection, coding, and analysis with theoretical sampling in order to generate theory that is integrated, close to the data, and expressed in a form clear enough for further testing" (Conrad, Neumann, Haworth, \& Scott, 1993). According to Glaser \& Strauss (1967) Constant comparative methodology incorporates four stages:

(1) Comparing incidents applicable to each category,

(2) Integrating categories and their properties,

(3) Delimiting the theory, and

(4) Writing the theory

Throughout the four stages of the constant comparative method, the researcher continually sorts through the data collection, analyzes and codes the information, and reinforces theory generation through the process of theoretical sampling. The benefit of using this method is that the research begins with raw data; through constant comparisons a substantive theory will emerge. Grounded theory is a laborintensive task that requires the researcher to invest time in the processes of analysis and data collection.

\section{Qualitative Data Analysis Process in Social Sciences}

Qualitative researches in Social Sciences interpret cultures and people's life-ways rather than seeking causal explanations for cultural practices. American anthropologist Clifford Geertz was a foremost figure of the interpretive or the symbolic anthropology school. According to the interpretive approach in anthropology, meaning is constituted out of interaction between individuals; the symbols and meanings are public in their significance and are not confined to single individual brains or the private sphere. It is the public or social aspects of meaning that is the focus of analysis for the proponents and followers of this approach. Geertz makes an influential case for anthropology as an interpretive science by clarifying that clarification of details in local cultural context rather than aiming at grand comparison is "interpretive anthropology" and also the way for anthropological work. For Geertz (1973), the concept of culture is fundamentally a semiotic one and he makes it clear in one of his most frequently quoted statements:

Believing with Max Weber, that man is an animal suspended in webs of significance he himself has spun, I take culture to be those webs, and the analysis of it to be therefore not an experimental science in search of law but an interpretive one in search of meaning.

Hence, interpretive approach discards attempts at causal explanations for cultural phenomena Geertz also used the metaphor of thick description which means the interpretation of all the minor details of human life in thick layers of contextual significance. According to Geertz "ethnography is thick description" and therefore his approach to culture is based on the metaphor of culture as text. Given this, an ethnographer's role is to interpret the text in a qualitative world - that is, reading cultures as if they were texts of some sort. The Gurung Arghau or Pae (After death rituals) and the Ghapren (Gurung priest) reciting the mantras may be an illustration. Based on this argument I bicker that qualitative analysis understands phenomenon and to go beyond reporting - move towards interpretation and identify themes and sub-themes. In the process of research, collection and analysis occur simultaneously and human-asan-instrument is the innermost point. Results can be used to investigate for further information and detail but the risk remains of diverting from research objectives. Nevertheless qualitative data analysis analyzes text, interview transcripts, photographs, art, field notes of observations etc. During the whole process of qualitative data analysis it is an admirable idea to engage in recording reflective notes about what we are learning from our data. The idea is to write memos (notes) when we have ideas and insights and to include those memos as additional data to be analyzed.

Several phases can be distinguished in qualitative data analysis process:

- $\quad$ Data cleaning

Initial data analysis (assessment of data quality)

- Main data analysis (answer the original research question)

- $\quad$ Final data analysis (necessary additional analyses and report)

Basic processes in qualitative data analysis serve a process of noticing, collecting and thinking. There is a simple foundation to the complex and rigorous practice of qualitative data analysis. Once we take hold of this foundation we can move about in many different directions. The consequence is the idea that Qualitative Data Analysis is a masterpiece based on three notes:

a. Noticing,

b. Collecting, and

c. Thinking about interesting things.

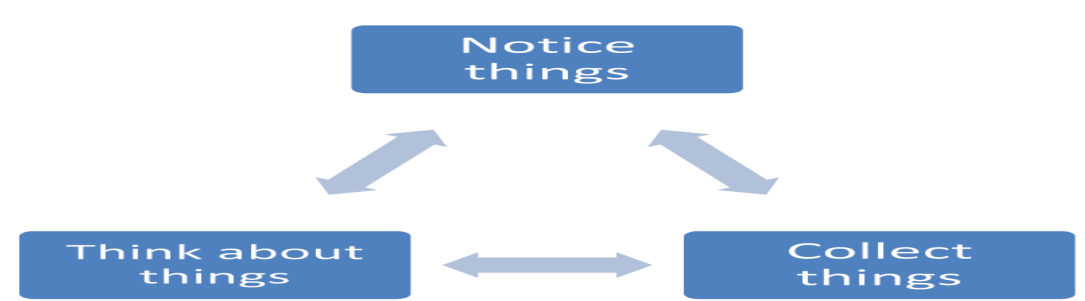

Source: Seidel, 1998

Qualitative data analysis process is not linear. When we do qualitative data analysis we do not simply Notice, Collect, and then Think about things, and then write a paper or report, rather, the process has the following characteristics (Seidel 1998):

a) Iterative and Progressive: The process is iterative and progressive as it is a cycle that keeps repeating. For example, when we are thinking about things we also start noticing new things in the data. We then collect and think about these new things. In principle the process is a never-ending twist (spiral).

b) Recursive: The process is recursive because one part can call us back to a previous part. For example, while we are busy collecting things we might simultaneously start noticing new things to collect.

c) Holographic: The process is holographic in that each step in the process contains the entire process. For example, when we first notice things you are already mentally collecting and thinking about those things. Hence, while there is a simple foundation to qualitative data analysis, the process of doing qualitative data analysis is complex. The key is to root researcher in this foundation and the rest will flow from this foundation. 
Order in Paradox: Myth, Ritual, and Exchange among Nepal's Tamang by David Holmberg (1989) incorporate -iterative and progressive, recursive, holographic characteristics. Holmberg conducted field work and his analysis is based on noticing, collecting and thinking. Holmberg's analysis is not only an ethnographic account but penetrates the Shaman/Sorcery Buddhism and traditions of sacrifice based complex cultural structure of the Tamangs. The analysis process is holographic in that each step in the process contains the entire process. The focus beyond the ethnographic is on the structure of a complex ritual field composed of Buddhist, Shamanic, and sacrificial practitioners and on the relation of this structure to the logics and contradictions of Tamang social exchanges, especially those associated with cross-cousin marriage. The role of symbolism remains fundamental in Tamang culture. Holmberg (1989) articulates;

Tamang symbology was characterized by a mythic closure which is related directly to a highly localized geography, society, polity, and economy. The world of Tamdungsa — the Tamang village where I resided -has now open up.

Holmberg squabbles that the political symbology which gave the local ritual substance has been translated in large measure into an idiom of kinship where kinship and polity are no longer coextensive as they have been in the past.

\section{Conclusion}

Qualitative research is the hallmark of social science research. Media revolution has revolutionized social science research. Expansion in mass communication, telecommunication, electronic media like frequency module (F.M.) radio television, media, and information technology have marvelous insinuation for social sciences-its concepts, theory, methods, field study/stay, analysis, practice, report writing etc. Interpretivist tradition in qualitative research is undergoing the alteration concerned with interpretation of social world, understanding of social world and experiences of social world. Social researchers heuristic traditions of asking Who? What? When? Where? Why? Seeing, Hearing and Reading from the people and places, events and activities are altering. Their learning about aspects of the social world for generating new understandings that can be used by that social world is under modification. The significance of grounded theory remains vital which holds the notion that theory could materialize through qualitative data analysis via the use of multiple stages of collecting, refining, and categorizing the data. Similarly the studied group may now be conversing, discoursing, raising questions, making remarks, doing arguments, giving and sharing opinions with logics with qualitative researcher straightforwardly or obliquely. Given this, the fundamental concern now is how are the existing social sciences concepts, theories and methods going to concentrate on different problems and issues appropriately and sufficiently in order to get an evocative and holistic image - which was once a hallmark of social sciences and still prevailing. However, in very speedily changing information dominated globalized world, innovative traditions of the discernment of emerging local and global contexts and versities need to be exposed and acknowledged as well as practiced in qualitative social science researches.

\section{References}

Barnard, A., Burgess, T. and Kirby, M. (2004). Sociology. Cambridge University Press.

Conrad, C., Neumann, A., Haworth, J. G., \& Scott, P. (1993). Qualitative research in higher education. Experiencing alternative perspective and approaches. Needham Heights, MA: Ginn Press.

Creswell, J. W. (2007). Research design: Qualitative and quantitative approaches. (2nd ed.). Thousand Oaks, CA: Sage.

Denzin, N. K., \& Lincoln, Y. S. (Eds.). (2005). Handbook of qualitative research. (3rd ed.). Thousand Oaks, CA: Sage.

Geertz, C. (1973). Thick Description: Toward an Interpretive Theory of Culture. The Interpretation of Cultures. New York: Basic Books.

Glaser, B. G., \& Strauss, A. L. (1967). The discovery of grounded theory: Strategies for qualitative research. Hawthorne, NY: Aldine.

Holmberg, D. (1989). Order in Paradox: Myth, Ritual, and Exchange among Nepal's Tamang. Ithaca and London: Cornell University Press.

Ortner, Sherry B. (1978). Sherpas through their Rituals. New York: Cambridge University Press.

Ortner, Sherry B. (1986). Theory in Anthropology since the Sixties. Comparative Studies in Society and History. London: Arnold Publication

Seidel, J. (1998). Method and Madness in the Application of Computer Technology to Qualitative Data Analysis. Using Computers in Qualitative Research. N. Fielding and R. Lee, eds., Newbury Park: Sage Publication.

Taylor, S. J., \& Bogdan, R. (1998). Introduction to qualitative research methods: A guidebook and resource. (3rd ed.). New York: Wiley.

Upadhyay, P. (2011). Research Methods in Sociology/Anthropology. Kathmandu, Khitiz publication. 